NASA/CR-1998-208713

ICASE Report No. 98-43

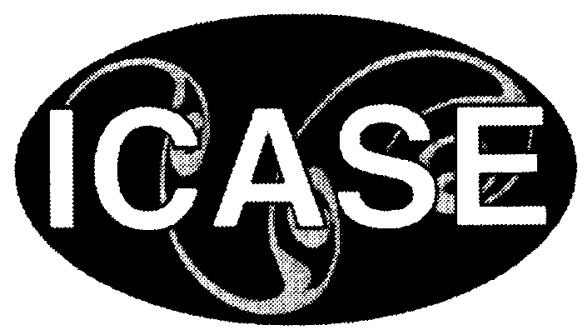

\title{
A Note on Kinetic Energy, Dissipation and Enstrophy
}

Jie-Zhi Wu

The University of Tennessee Space Institute, Tullahoma, Tennessee

Ye Zhou

ICASE, Hampton, Virginia and

IBM, Yorktown Heights, New York

Meng Fan

The University of Tennessee Space Institute, Tullahoma, Tennessee

Institute for Computer Applications in Science and Engineering

NASA Langley Research Center

Hampton, VA

Operated by Universities Space Research Association

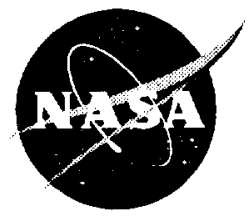

National Aeronautics and

Space Administration

Langley Research Center

Hampton, Virginia 23681-2199 
Available from the following:

NASA Center for AeroSpace Information (CASI)

7121 Standard Drive

Hanover, MD 21076-1320

(301) 621-0390
National Technical Information Service (NTIS) 5285 Port Royal Road

Springfield, VA 2216 - 2171

(703) $487-4650$ 


\title{
A NOTE ON KINETIC ENERGY, DISSIPATION AND ENSTROPHY*
}

\author{
JIE-ZHI WU ${ }^{\dagger}$, YE ZHOU ${ }^{\ddagger}$, AND MENG FAN $\S$
}

\begin{abstract}
The dissipation rate of a Newtonian fluid with constant shear viscosity can be shown to include three constituents: dilatation, vorticity, and surface strain. The last one is found to make no contributions to the change of kinetic energy. These dissipation constituents are used to identify typical compact turbulent flow structures at high Reynolds numbers. The incompressible version of the simplified kinetic-energy equation is then cast to a novel form, which is free from the work rate done by surface stresses but in which the full dissipation reenters.
\end{abstract}

Key words. kinetic energy, dissipation, enstrophy, surface strain

Subject classification. Fluid Mechanics

1. Introduction. We make a general theoretical examination on the relation between kinetic energy transport, dissipation, and enstrophy. First, we derive an exact but simplified transport equation for the kinetic energy of viscous compressible flow, in which the full dissipation function is replaced by the squares of vorticity and dilatation. This forms a local counterpart of the classic Bobyleff-Forsyth formula [1] on the integral equivalence of incompressible dissipation and enstrophy under special boundary condition, and provides a physical clarification on dynamic processes really involved in the evolution of kinctic cnergy. The dissipation constituents are used to identify typical compact flow structures. Sccond, we cast the incompressible version of the simplified kinetic-energy equation to a novel form, of which the local spatical average does not explicitly depend on boundary conditions, but the full dissipation reappears along with enstrophy. This result is of relevance to some current studies of intermittency and scaling laws in inhomogencous and anisotropic turbulence.

2. Dissipation constituents. Let $\boldsymbol{\omega}=\nabla \times \boldsymbol{u}$ and $\vartheta=\nabla \cdot \boldsymbol{u}$ be the vorticity and dilatation, $\mathbf{S}$ and $\mathbf{T}$ be the strain-rate and stress tensor, respectively, so that for Newtonian fluid $\mathbf{T}=(-p+\lambda \vartheta) \mathbf{I}+2 \mu \mathbf{S}$ (c.g. $[1,2])$, where $I$ is the unit tensor, and $\mu$ and $\lambda$ are the shear and second viscosity ${ }^{1}$. Throughout this note we assume $\mu$ is constant. The conventional transport equation for kinetic encrgy $E=|\boldsymbol{u}|^{2} / 2$ reads

$$
\rho \frac{D E}{D t}=\rho f \cdot \boldsymbol{u}+\vartheta p+\nabla \cdot(\mathbf{T} \cdot \boldsymbol{u})-\Phi
$$

* The work was supported in part by ONR under the Grant W00014-96-1-0452, and by the National Aeronautics and Space Administration under NASA Contract No. NAS1-97046 while the second author was in residence at the Institute for Computer Applications in Science and Engineering (ICASE), NASA Langley Research Center, Hampton, VA 23681-2199. The third author was also supported by the J.M. Wu research Fund of UTSI.

$\dagger$ The University of Tennessee Space Institute, Tullahoma, Tennessee, 37388

‡ IBM Research Division, T. J. Watson Research Center, Yorktown Heights, NY 10598 and Institute for Computer Applications in Scicnce and Engineering, NASA Langley Research Center, Hampton, VA 23681

$\$$ The University of Tennessec Space Institute, Tullahoma, Tennessec, 37388; On leave from State Key Laboratory on Turbulence Research, Peking University, Beijing 100871, China

${ }^{1}$ One may also replace $\mathbf{S}$ by its derivatoric part $\mathbf{S}^{\prime}=\mathbf{S}-\frac{1}{3} \vartheta \mathrm{I}$ so that $\mathrm{T}=(-\boldsymbol{p}+\zeta \vartheta) \mathbf{I}+2 \mu \mathbf{S}^{\prime}$, where $\zeta=\lambda+\frac{2}{3} \mu$ is the bulk viscosity coefficient. This does not affect our argument but is less convenient. 
where $f$ is a body force and $\Phi=\lambda \vartheta^{2}+2 \mu \mathbf{S}: \mathbf{S}$ is the dissipation ratc. It is straightforward to derive an invariant decomposition $\Phi=\widehat{\Phi}+\Phi_{B}$, where

$$
\begin{gathered}
\widehat{\Phi}=(\lambda+2 \mu) \vartheta^{2}+\mu \mid \boldsymbol{\omega}^{2}, \\
\Phi_{B}=-\nabla \cdot(2 \mu \mathbf{B} \cdot \boldsymbol{u}),
\end{gathered}
$$

are, respectively, a positive-definite part caused by $\vartheta^{2}$ and $|\omega|^{2}$, and a non positive-definite "dissipation" caused by surface-strain rate tensor ${ }^{2}[3]$

$$
\mathbf{B} \equiv \vartheta \mathbf{I}-\nabla \boldsymbol{u}^{T} \quad \text { with } \quad \nabla \cdot \mathbf{B}=\mathbf{0} .
$$

From (2) the compressible extension of the Bobyleff-Forsyth formula follows:

$$
\int_{V} \Phi d V=\int_{V}\left[(\lambda+2 \mu) \vartheta^{2}+\mu|\omega|^{2}\right] d V-2 \mu \int_{\partial V} n \cdot \mathbf{B} \cdot \boldsymbol{u} d S
$$

Thus, if $\boldsymbol{n} \cdot(\mathbf{B} \cdot \boldsymbol{u})=\mathbf{0}$ on $\partial V$, we have an integral equivalence of $\boldsymbol{\Phi}$ and $\widehat{\Phi}$.

A similar decomposition of $\mathbf{T}$ has been introduced by $\mathrm{Wu}$ and $\mathrm{Wu}[4-5]$ :

$$
\mathbf{T}=\widehat{\mathbf{T}}+\mathbf{T}_{B}, \quad \widehat{\mathbf{T}} \equiv-\Pi \mathbf{I}+2 \mu \boldsymbol{\Omega}, \quad \mathbf{T}_{B} \equiv-2 \mu \mathbf{B},
$$

where $\Pi \equiv p-(\lambda+2 \mu) \vartheta$ is the isotropic part of $\mathbf{T}$ and $\boldsymbol{\Omega}$ is the vorticity tensor. Substituting (5) into the Cauchy motion equation reveals that $\mathbf{T}_{B}$ plays no role in momentum balance. Now, substituting (5) into $\nabla \cdot(\mathbf{T} \cdot \boldsymbol{u})$ in (1) leads to a splitting of the work rate $W$ per unit volume done by surface stress:

$$
W=\nabla \cdot(\mathbf{T} \cdot \boldsymbol{u})=W_{p}+W_{\vartheta}+W_{\omega}+W_{B}
$$

where

$$
\begin{gathered}
W_{p}=-\nabla \cdot(p \boldsymbol{u}), \quad W_{\vartheta}=\nabla \cdot[(\wedge+2 \mu) \vartheta \boldsymbol{u}], \\
W_{\omega}=-\nabla \cdot(\mu \boldsymbol{\omega} \times \boldsymbol{u}), \quad W_{B}=-\nabla \cdot(2 \mu \mathbf{B} \cdot \boldsymbol{u}) .
\end{gathered}
$$

Thus, $W_{B}$ and $\Phi_{B}$ are always self-balanced, making no contribution to the change of $E$. Hence, Eq. (1) can be simplified to

$$
\rho \frac{D E}{D t}=\rho \boldsymbol{f} \cdot \boldsymbol{u}+\vartheta p+\nabla \cdot(\widehat{\mathbf{T}} \cdot \boldsymbol{u})-\widehat{\Phi},
$$

which proves a local "equivalence" of $\Phi$ and $\widehat{\Phi}$ under the conditi on that $\mathbf{T}$ be replaced by $\widehat{\mathbf{T}}$ simultaneously. Whenever $\mathbf{T}_{B}$ does a work (positive or negative), it is directly and locally dissipated to $\Phi_{B}$ which, as is easily proved, does cause a change of the internal energy. The refore, so far as its mechanical aspects are concerned, a Newtonian fluid can well be simplified to a hypoth tic medium of which the motion consists of only an isotropic expansion/compression and a spin. A significant saving of this simplification in numerical computation was cited in [5]. The surface deformation, which is generically most complicated, plays no role except on free surface [4].

\footnotetext{
${ }^{2}$ In this report and Dishington's paper there is a sign difference in the clefinition of $\mathbf{B}$.
} 
In the above analysis a key issue is the distinction between $\widehat{\Phi}$ and $\Phi_{B}$ or the relation between $\Phi$ and $\widehat{\Phi}$. Studying their distribution characters allows us to identify different coherent and compact structures in high Reynolds-number flows. Typically, these include two shearing structures: thin vortex layers and tubes, and a compressing structure, shock waves. While across a shock there is evidently an extremely strong peak of $\vartheta^{2}$, the distribution character of $\omega^{2}$ and $\Phi_{B}$ for shearing structures can be casily illustrated by idealized models. For a unidirectional shear flow with $u=(U(y, t), 0,0)$ in Cartesian coordinates $(x, y, z)$, there is

$$
\widehat{\Phi}=\Phi=\mu \omega^{2}=\left(\frac{\partial U}{\partial y}\right)^{2}, \quad \Phi_{B}=0
$$

while for a pure vortex with $\boldsymbol{u}=(0, v(r, t), 0)$ and $\boldsymbol{\omega}=(0,0, \omega(r, t))$ in cylindrical coordinates $(r, \theta, z)$, there is

$$
\widehat{\Phi}=\mu \omega^{2}, \quad \Phi_{B}=W_{B}=-4 \mu \frac{v}{r} \frac{\partial v}{\partial r}, \quad \Phi=\mu\left(\frac{\partial v}{\partial r}-\frac{v}{r}\right)^{2}
$$

Note that inside the vortex core $\left(r<r_{0}\right)$ with $\partial v / \partial r>0$ there is a negative peak of $\Phi_{B}$ which cancels the peak of $\widehat{\Phi}\left(\widehat{\Phi}=\widehat{\Phi}_{\max }\right.$ at $\left.r=0\right)$, so that $\Phi$ remains small as it should ( $\Phi=0$ in a solid-like core). But outside the core $\Phi_{B}$ reaches a hollow-tube like (macaroni-likc) positive peak $\Phi_{B \max }$ at an $r=r_{m}>r_{0}$, which becomes the main contributor to $\Phi$. For $r>r_{m}$ the contribution of enstrophy to $\Phi$ is neglegible. The peak behaviors of $\widehat{\Phi}$ and $\Phi_{B}$ for layers and tubes can be easily checked by considering, say, $U(y)=\tanh (y / \delta)$ with $\delta \ll 1$ and an Oseen vortex with $\mu \ll 1$, respectively.

When $R e \gg 1$, these idealized models can serve as local building blocks of more generic layer and tube structures, provided that the strain components along the layers or tube axes are weaker than the vorticity therein. In particular, in a compressible turbulence the strong peaks of $\vartheta^{2}, \omega^{2}$, and the simultaneous peaks of $\omega^{2}$ and $\Phi$ are good indicators of random shocklets, filaments, and shearing shects, respectively. Indeed, in a numerical simulation of two-dimensional supersonic turbulence, Porter et al. [6] have observed complex sheetlike shocklet structures by visualizing dilatation field. From their direct numerical simulations of a uniformly sheared incompressible turbulence, Tanaka and Kida [7] used the ratio $\widehat{\Phi} / \Phi$ to identify many thin vortex filaments with $\widehat{\Phi} / \Phi>2$ (as shown above, these high-enstrophy structures must be surrounded by weaker hollow-tube peaks of strain), while at the high enstrophy region with comparable strain $1 / 2<\widehat{\Phi} / \Phi<4 / 3$, the vortex sheet structures were observed. These results are confirmed by Boratav and Pelz [8], who conducted direct numerical simulations of an unforced flow and investigated the strain-vorticity correlation in a plane spanned by $\Phi / \mu$ and enstrophy $\Phi / \mu$. Now, our result provides a theoretical support for these identifications. Interestingly, for the Oseen vortex at $t=1$ we found $\Phi_{B \max } / \widehat{\Phi}_{\max }=0.09$, completely independent of the Reynolds number. The ratio $r_{m} / r_{0}$, which is about 1.2 , has only a very weak dependence on $R e$. Thus, as $R e \rightarrow \infty$ and $r_{0} \rightarrow 0$, the distribution of $\widehat{\Phi}$ and $\Phi_{B}$ (or the full $\Phi$ ) for a filament becomes a singular "spaghetti" enclosed by a singular "macaroni", having the same fractal dimension (approaching 1).

3. Kinetic-energy equation. We now further focus on incompressible flow with $\rho=1$, which is a typical situation where the thermodynamics is not involved. In this case (6) reduces to

$$
\frac{D E}{D t}=\boldsymbol{f} \cdot \boldsymbol{u}-\nabla \cdot(p \boldsymbol{u})-\nu \nabla \cdot(\boldsymbol{\omega} \times \boldsymbol{u})-\widehat{\Phi} .
$$

In the studies of turbulent-energy budget one removes the divergence terms in (7) by taking average over a region with periodic boundary conditions. Then for statistically steady turbulence and as $\nu \rightarrow 0$, one obtains a balance solely between the work of stiring force $f$ and energy cascade in integral range, and a balance solely 
between the cnergy cascade and dissipation (enstrophy) in ines tial range (e.g. Frisch [9]). This procedure is necessary to obtain the Kolmogorov scaling law and its mode rn revisions. However, for the local scaling of structure functions in an inhomogencous and anisotropic tursulence, the boundary condition on a small sphere (in which the average is to be taken) must be kept generic. Therefore, it will be highly interesting if a general incompressible energy equation can be cast to a form free from work-rate terms, so that the averaging process does not explicitly rely on specific boundary conditions. We now consider this issue.

The advection of $E$ and pressure work in (7) can be combined to $\nabla \cdot\left(u h_{0}\right)$, where $h_{0}$ is the stagnation enthalpy. To remove this term we split the momentum equation into two parts: a transverse or divergence-free part (including harmonic components), and a longitudinal or :url-free part. Namely, we make a decomposition of $\boldsymbol{f}$ and the Lamb vector $\boldsymbol{l}=\boldsymbol{\omega} \times \boldsymbol{u}$, denoted by $\boldsymbol{f}=\boldsymbol{f}_{\perp}+\boldsymbol{f}_{\|}$and $\boldsymbol{l}=\boldsymbol{l}_{\perp}+\boldsymbol{l}_{\|}$. It then follows that

$$
\frac{\partial \boldsymbol{u}}{\partial t}=\boldsymbol{f}_{\perp}-\boldsymbol{l}_{\perp}-\nu \nabla \times \boldsymbol{\omega}, \quad \nabla h_{0}=-\boldsymbol{l}_{\|},
$$

where $\boldsymbol{l}_{\perp}$ is the projection of advection $\boldsymbol{u} \cdot \nabla \boldsymbol{u}$ onto the solenoidal space. From (8a) there is

$$
\frac{\partial E}{\partial t}=\left(\boldsymbol{f}_{\perp}-\boldsymbol{l}_{\perp}\right) \cdot \boldsymbol{u}-\nu \nabla \cdot \boldsymbol{l}-\widehat{\boldsymbol{\Phi}}
$$

Compared to (7), the pressure work is absent, and advection retains a "residue" $\boldsymbol{l}_{\perp} \cdot \boldsymbol{u}$, which is implicitly boundary-dependent since the projection is a global operator. Then, since

$$
\widehat{\Phi}=\nu|\boldsymbol{\omega}|^{2}, \quad \Phi_{B}=2 \nu \nabla \cdot(\boldsymbol{u} \cdot \nabla \boldsymbol{u})=-2 \nu \nabla^{2} p,
$$

there is

$$
-\nu \nabla \cdot \boldsymbol{l}=\nu \nabla^{2} h_{0}=\nu \nabla^{2} E-\frac{3}{2}(\Phi-\widehat{\Phi}) .
$$

Therefore, from (9) a novel diffusion equation for the kinetic energy follows:

$$
\left(\frac{\partial}{\partial t}-\nu \nabla^{2}\right) E=\left(f_{\perp}-l_{\perp}\right) \cdot u-\frac{1}{2}(\Phi+\widehat{\Phi})
$$

in which dissipation and enstrophy appear simultaneously and symmetrically, but all boundary-dependent work-rate terms disappear. Note that the reappearance of $\Phi$ in (10) does not conflict with (7); it comes from a part of the vorticity work (the other part being the diffusion o: $E$ ). Morcover, compared to the usual form of the energy cascade [9], we now see that the cascade is neatly 1 epresented by the statistic average of $\boldsymbol{l}_{\perp} \cdot \boldsymbol{u}$, which is dominated by the transverse Lamb vector and has a compact support where $\boldsymbol{l}_{\perp} \neq 0$.

4. Conclusion. It has been noticed that the relative co atributions to the dissipation from straindominated and enstrophy-dominated structures may be relevast to the strength of intermittency, as well as to the scalings in longitudinal and transversal structure fur ctions [10]. Now, by placing the enstropy and dissipation in equal footing and exhibiting their interplay, Eq. (10) clearly emphasizes that, if a more boundary-independent (and hence more universal) theory is to ke reached, both strain rate (via dissipation) and vorticity (via enstrophy) are important in the energy tras sfer process. On the other hand, if (7) is used, one need reconsider the respective roles of full dissipation ind enstrophy. In either case a more careful treatment of the effect of boundary condition on the average sver a small sphere in inhomogencous and anisotropic turbulence is necessary. 
Public reporting burden for this collection of information is estimated to average 1 hour per response, including the time for reviewing instructions, searching existing data sources, gathering and maintaining the data needed, and completing and reviewing the collection of information. Send comments regarding this burden estimate or any other aspect of this Davis Highway, Suite 1204. Ariington, VA 22202-4302, and to the Office of Management and Budget, Paperwork Reduction Project (0704-0188), Washington, DC 20503.

\begin{tabular}{|l|l|l}
\hline 1. AGENCY USE ONLY(Leave blank) & $\begin{array}{c}\text { 2. REPORT DATE } \\
\text { September } 1998\end{array}$ & $\begin{array}{l}\text { 3. REPORT TYPE AND DATES COVERED } \\
\text { Contractor Report }\end{array}$ \\
\hline
\end{tabular}

4. TITLE AND SUBTITLE

A note on kinctic encrgy, dissipation and enstrophy

5. FUNDING NUMBERS

C NAS1-97046

WU 505-90-52-01

6. AUTHOR(S)

Jie-Zhi Wu

Ye Zhou

Meng Fan

7. PERforming ORgANizATION NAME(S) AND ADDRESS(ES)

Institute for Computer Applications in Science and Enginecring

Mail Stop 403, NASA Langley Research Center

Hampton, VA 23681-2199

9. SPONSORING/MONITORING AGENCY NAME(S) AND ADDRESS(ES)

National Aeronautics and Space Administration

Langley Research Center

Hampton, VA 23681-2199
8. PERFORMING ORGANIZATION REPORT NUMBER

ICASE Report No. $98-43$

\section{SUPPLEMENTARY NOTES}

Langley Technical Monitor: Dennis M. Bushnell

Final Report

Submitted to Physics of Fluids

12a. DISTRIBUTION/AVAILABILITY STATEMENT

12b. DISTRIBUTION CODE

Unclassified Unlimited

Subject Category 34

Distribution: Nonstandard

Availability: NASA-CASI (301)621-0390

10. SPONSORING/MONITORING

AGENCY REPORT NUMBER

NASA/CR-1998-208713

ICASE Report No. 98-43

13. ABSTRACT (Maximum 200 words)

The dissipation rate of a Newtonian fluid with constant shear viscosity can be shown to include three constituents: dilatation, vorticity, and surface strain. The last one is found to make no contributions to the change of kinetic energy. These dissipation constituents are used to identify typical compact turbulent flow structures at high Reynolds numbers. The incompressible version of the simplified kinetic-energy equation is then cast to a novel form, which is frec from the work rate done by surface stresses but in which the full dissipation reenters.

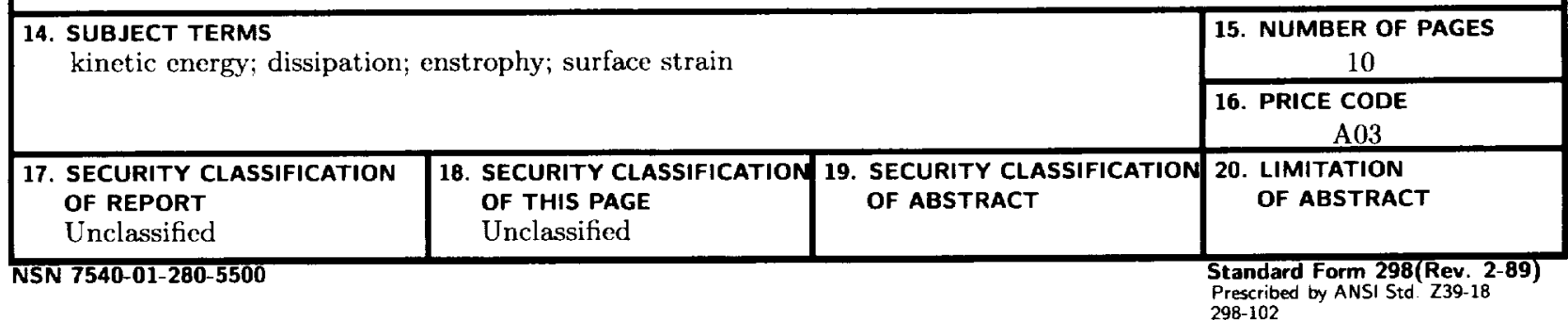




\section{REFERENCES}

[1] J. Serrin, "Mathematical principles of classic fluid mechanics," Handbuch der Physik VIII.1, ed. by S. Flügge, Springer-Verlag, (1959).

[2] P.A. Lagerstrom, Laminar Flow Theory, Princeton Univ. Press, (1964).

[3] R.H. Dishington, "Rate of surface strain," Am. J. Phys. 33, (1965), p. 827.

[4] J.Z. Wu, "A theory of three-dimensional interfacial vorticity dynamics," Phys. Fluids 7, (1995), pp. 2375-2395.

[5] J.Z. Wu And J.M. Wu, "Vorticity dynamics on boundaries," Adv. Appl. Mech. 32, (1996), pp. 113-269.

[6] D.H. Porter, A. Pouquet, And P.R. Woodward, "A numerical study of supersonic turbulence," Theoret. Comput. Fluid Dynamics 4, (1992), pp. 13-49.

[7] M. Tanaka And S. Kida, "Characterization of vortex tubes and sheets," Phys. Fluids A5, (1993), p. 2079.

[8] O.N. Boratav And R.B. Pelz, "Structures and structure functions in the inertial range of turbulence," Phys. Fluids 9, (1997), p. 1400.

[9] U. Frisch, Turbulence, Cambridge Univ. Press, (1995).

[10] G. He, S. Chen, R.H. Kraichnan, R. Zhang, and Y. Zhou, "Statistics of Dissipation and Enstrophy Induced by a Set of Burgers Vortices," Submitted to Phys. Rev. Lett., (1998). 